\title{
Estimación de la ventilación y el ruido en el metro de la Ciudad de México
}

\author{
O.R. San Román-Gutiérrez \\ División de Ciencias Básicas, Facultad de Ingeniería, UNAM \\ E-mail: sanroma320@ hotmail.com
}

(recibido: febrero de 2003; aceptado: abril de 2003)

\section{Resumen}

Se presentan los resultados de las mediciones de velocidad de viento y ruido realizadas en trenes de las líneas 1 y 3 del Sistema Colectivo Metro de la Ciudad de México. En los trenes de mayor antigüedad se observó una ventilación prácticamente inútil para los pasajeros, en tanto que los vagones más modernos de la línea 1 cuentan con una ventilación aceptable; sin embargo, se presentan grandes variaciones a lo largo del vagón. Se encontró un alto número de ventiladores desbalanceados y un promedio de dos ventiladores por vagón fuera de operación.

Se detectó que el problema prin cipal radica en la extracción del aire, y no en el suministro, por lo que se propone que los diseños futuros incluyan la extracción efectiva y la circulación adecuada del aire.

El ruido no rebasa las normas, pero resulta molesto, pues alcanza los $80 \mathrm{~dB}$ a la altura media de los pasajeros, con una variación entre el centro y el extremo del vagón de aproximadamente $5 \mathrm{~dB}$, sin incluir un incremento de entre 5 a $7 \mathrm{~dB}$ debido a los vendedores.

Descriptores: ventilación, ruido, comodidad, metro.

\begin{abstract}
Wind velocity and noisemea surements in lines 1 and 3 of M exico's $s$ ity M etro subway arepresented. Whilein old units theven ti la tion is al mostnull, in mod ern trains itisacceptable. A largenum ber of fans were detected un bal anced, and an aver age of two fans per waggon were out of or der. Largewind vari a tions along the waggons were pres ent. It was detected that themain prob lem was due to an unadequate ex traction and cir cu la tion of the air and not due to the sup ply. Therefore, it is pro posed that a redesign should be un der taken for thefu ture.

Thenoise com plies with the mex $i$ can stan dard, but it reaches $80 \mathrm{~dB}$ and it is an noy ing for the passangers. Thereis a $5 \mathrm{~dB}$ vari a tion between the cen ter and the ex treme of the waggon, and a 5 to $7 \mathrm{~dB}$ raise due to the loudly voices of "sales per sons".
\end{abstract}

Keywords: ven tila tion, noise, com fort, subway. 


\section{Objetivo}

Estimar las condiciones de comodidad en las líneas 1 y 3 del metro de la Ciudad de México, con base en mediciones de velocidad de viento $\mathrm{y}$ de ruido en el in te rior de los vagones, que permitan futuros diseños con una extracción y circulación adecuada del aire.

\section{Introducción}

Uno de los problemas que afectan públicamente al "Sistema de Transporte Colectivo Metro" es su inadecuada ventilación, tarea que dista de ser simple. No obstante, debe reconocerse que en las unidades más recientes ha sido mejorada, no así en los carros de mayor antigüedad, donde con una ventilación insuficiente, el viaje resulta incómodo. En este trabajo se presentan los resultados de las mediciones de velocidad de viento y de ruido efectuadas en vagones de las líneas 1 y 3.

\section{Las cargas de calor y la comodidad de los usuarios}

Se considera que en cada vagón se transportan aproximadamente de 200 a 249 perso nas du rante las horas pico, lo cual gen era calor y aro mas que desagradan a los usuarios. Estas cargas de calor, sumadas a las del tren y del ambiente que se infiltran al vagón, constituyen la carga de enfriamiento total que debe proporcionarse para mantener la comodidad de los usuarios.

La carga total de enfriamiento es complicada de establecer, debido a que está sujeta al calor generado por la fricción del sistema de frenos y al efecto de "almacenamiento de calor" o inercia térmica que se produce en los túneles de concreto. Estos pueden llegar a tener una masa de unas 20 toneladas por metro de túnel, y con un calor específico de $0.2 \mathrm{kcal} / \mathrm{kg}^{\circ} \mathrm{C}$, almacenan suficiente "calor" para mantener la temperatura alta después de varias horas de que el ambiente alcanzó su máximo, a pesar de las grandes cantidades de aire que se introducen a través del sistema de ventilación forzada.

El frenado hace que los motores eléctricos generen energía, que en los trenes de mayor antigüedad, se disipa mediante resistencias eléctricas, que a su vez son enfriadas por ventiladores que también generan calor. Otras contribuciones, como la del alumbrado interno y la de los ventiladores, son despreciables en comparación con la contribución de los pasajeros.

En este trabajo sólo se considerará la contribución de los pasajeros, ya que al parecer, los cálculos realizados para el diseño de la ventilación tomaron en cuenta solamente esta carga. Para la Ciudad de México la temperatura de confort recomendada para el verano se sitúa en $25^{\circ} \mathrm{C}$, con un margen habitual de $1^{\circ} \mathrm{C}$, en tanto que para invierno es de $20^{\circ} \mathrm{C}$, y suele variar entre 18 y $21^{\circ} \mathrm{C}$.

Los valores de humedad relativa entre los que puede oscilar este parámetro, se sitúan entre el 30 y el 65\%; sin embargo, una variación porcentual de unos pocos puntos puede conllevar cambios considerables en la comodidad, de ahí que es importante enfatizar la necesidad de que se tome en cuenta, ya que una alta humedad producirá la sudoración excesiva en los pasajeros, con el consecuente malestar in di vid ual y gen eral.

También debe tomarse en cuenta que la atmósfera de un lugar cerrado puede parecer pesada debido a la ausencia de variación en las corrientes de aire. La sensación de frescura se percibe con los órganos sensoriales de la 
piel, los cuales reaccionan a cambios de temperatura y de las corrientes. Si la velocidad del aire y la temperatura son constantes, no hay variación en la atmósfera para estimular las reacciones físicas. Las corrientes de aire vari ables causan cambios momentáneos en la temperatura de la piel, dando sensación de frescura, que resulta en un efecto tanto psicológico como fisiológico. No obstante que no haya cambio de temperatura, el resultado final es que las variaciones de las corrientes de aire hacen parecer un sitio más fresco.

Para el diseño del metro se consideró una carga de diez personas $/ \mathrm{m}^{2}$ de pie y 39 personas sentadas. Siguiendo las recomendaciones de Carrier (1987) para condiciones similares (sólo en cuanto a número de ocupantes por metro cuadrado, ya que esta referencia no considera las condiciones para trenes urbanos), se estableció un caudal volumétrico de aire entre 30 y 50 pies $3 / \mathrm{min} /$ persona (51 a $85 \mathrm{~m}^{3} / \mathrm{h} /$ persona). Si se considera el flujo mínimo, se requieren:

$$
\begin{aligned}
V_{\min } & =(249 \text { personas }) *\left(51 \mathrm{~m}^{3} / \mathrm{h} / \text { persona }\right) \\
& \sim 12,700 \mathrm{~m}^{3} / \mathrm{h}<13,000 \mathrm{~m}^{3} / \mathrm{h}
\end{aligned}
$$

Para esta tarea se instalan siete ventiladores en el techo de cada vagón. Según se reporta en las pruebas de laboratorio 0 de banco, cada ventilador puede dar un gasto de $3200 \mathrm{~m}^{3} / \mathrm{h}$, por lo que se garantizaría un suministro mínimo de 1,800 a 2,000 m³/h, es decir, de $14,000 \mathrm{~m} 3 / \mathrm{h}$ con una velocidad recomendada de 0.4 a $1.5 \mathrm{~m} / \mathrm{s},(1,440$ a 5,400 $\mathrm{m} / \mathrm{h}$ ). Aunque no se tienen las cargas específicas del calor promedio generado en el metro, éstas pueden estimarse a partir de las tablas de generación de calor para personas realizando diversas actividades del ASHRAE (1993) (Cuadro 1).

Para los cálculos de este trabajo se usará una carga de enfriamiento de $100 \mathrm{~W} /$ persona

Dado lo anterior, podría suponerse que los problemas de ventilación fueran mínimos; sin embargo, esto no es así, por lo menos en los vagones con mayor antigüedad.

Una alternativa para este cálculo se resume en Thermal Comfort (Kuisgard, 1997), En esta referencia se explican los índices que comúnmente se utilizan para estimar la comodidad de las personas, como el "Voto Predictivo Promedio" (PMV) y el Porcentaje de Personas Insatisfechas (PPD). Estos índices se obtienen a partir de la actividad realizada por las personas, la ropa que visten ("índice de CLO"), el tiempo de permanencia en el sitio y las características del ambiente, como: temperatura de bulbo seco, humedad relativa, velocidad de viento, temperatura de las paredes radiantes, latitud del lugar, entre otros. Con estos datos se evalúan las temperaturas efectiva y efectiva corregida, que se utilizan para el cálculo de las cargas de calor y de los índices de comodidad.

Cuadro 1

\begin{tabular}{lcccc}
\hline & Hombre & Calor ajustado (W)* & Calor sensible (W) & Calor latente (W) \\
\hline Sentado en reposo & 115 & 100 & 60 & 40 \\
Trabajo ligero sentado & 140 & 120 & 65 & 55 \\
Parado, caminando despacio & 235 & 150 & 75 & 75 \\
\hline
\end{tabular}

* calor ajustado, se refiere a una carga promedio formada por hom bres, mujeres y niños 
EI PMV establece una escala desde -3 (muy frío) hasta +3 (muy caliente), siendo cero el valor de comodidad óptima, con un margen de \pm 0.5 .

El índice PPD es más utilizado, pues mide directamente el porcentaje de personas insatisfechas. La norma establece que para una comodidad aceptable el PPD no debe exceder del $10 \%$ del total de personas. El cálculo de estos índices puede hacerse rápidamente con la herramienta que proporciona De Dear (2001).

\section{Evaluaciones realizadas}

Se efectuaron dos tipos de evaluación: a) En los talleres del metro, en un vagón de un tren en reposo con las ventanillas cerradas, y b) En condiciones de operación. Las mediciones se efectuaron en tres trenes de la línea 3 y en seis de la línea 1 (tres trenes modernos y tres antiguos).

En todos los casos se utilizó un anemómetro de hilo caliente con $2 \%$ de incertidumbre para medir las velocidades en la dirección del viento máximo. Se hacer notar que las velocidades reportadas, sólo son indicativas del orden de magnitud de la velocidad del viento; de ninguna manera se afirma que se hayan medido de acuerdo con normas.

\section{Evaluación en los talleres del metro}

\author{
Velocidad de viento
}

Se tomó al azar un vagón en reposo, vacío y con las ventanillas cerradas, posteriormente se midieron las velocidades de viento en los puntos cercanos a un ventilador (tomado al azar) a diferentes distancias en la horizontal y la vertical. Además se midió la velocidad de viento en las rejillas de succión y de evacuación. Los resultados (Tabla 1, figura 1) muestran que en la zona cercana al ventilador la velocidad del viento a la altura de las cabezas de los pasajeros de pie, es de 0.1 a $0.12 \mathrm{~m} / \mathrm{s}$, en tanto que en la zona lejana al ventilador, son prácticamente nulas.

La velocidad de viento a la salida de uno de los ventiladores, se midió en 36 nodos de una malla cuadrada de $12 \mathrm{~cm} \times 12 \mathrm{~cm}$, obteniéndose los resultados de la tabla $2 a$ y $2 b$. De las mediciones realizadas quedó claro que el ventilador evaluado presenta un marcado desbalanceo, pero el efecto más importante es la baja velocidad a la altura de los pasajeros de pie y la nula velocidad a la altura de los pasajeros sentados. Es de esperarse que bajo estas condiciones, el ventilador no satisfaga lo propuesto en el diseño.

Tabla 1. Velocidad de viento, vagón cerrado, vacío y en reposo

\begin{tabular}{|c|c|c|c|c|c|}
\hline $\begin{array}{l}\text { Zona de medición } \\
\mathrm{T}_{\text {ambiente }}=22^{\circ} \mathrm{C}\end{array}$ & $\underset{(\mathrm{cm})}{\operatorname{Distancia~al~techo~}}$ & $\begin{array}{c}\text { Vel. Promedio } \\
\text { (m/s) entre el } \\
\text { centro y } 0.72 \mathrm{~m}\end{array}$ & $\begin{array}{l}\text { Vel. Promedio } \\
\text { (m/s) entre } 0.72 \text { y } \\
1 \mathrm{~m} \text { (medido a } \\
\text { partir del centro) }\end{array}$ & $\underset{(\mathrm{m} / \mathrm{s})}{\text { Velocidad máxima }}$ & $\begin{array}{l}\text { Velocidad mínima } \\
\mathrm{m} / \mathrm{s})\end{array}$ \\
\hline & 10 & 0.42 & 0.1 & 1.3 & 0.0 \\
\hline & 32 & 0.4 & 0.36 & 0.7 & 0.01 \\
\hline Pasajeros de pie & 70 & 0.1 & 0.12 & 0.7 & 0.0 \\
\hline Pasajeros sentados & 94 & 0.09 & 0.0 & 0.2 & 0.0 \\
\hline
\end{tabular}


O.R. San Román-Gutiérrez

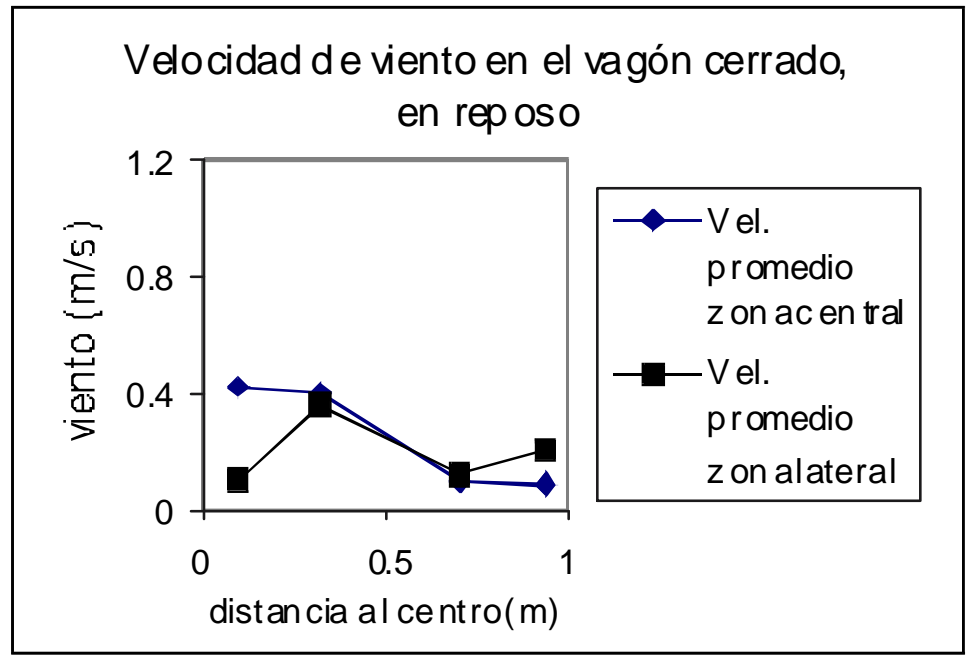

Figura 1

Tabla 2 a. Velocidad de viento $(\mathrm{m} / \mathrm{s})$ de un ventilador, vagón vacío, en reposo, ventanillas cerradas (distancia al techo: $0.10 \mathrm{~m}$ )

\begin{tabular}{cc}
\hline Distancia al centro del ventilador $(\mathrm{m})$ & Velocidades máximas $(\mathrm{m} / \mathrm{s})$ \\
\hline 0.06 & 0.46 \\
0.18 & $2.4 \pm 1.7$ \\
0.30 & $0.35+0.19$ \\
\hline
\end{tabular}

Tabla $2 \mathrm{~b}$. Velocidades horizontal y vertical $(\mathrm{m} / \mathrm{s}$ ) a $10 \mathrm{~cm}$ del techo (36 nodos de $12 \mathrm{~cm}$ de lado) con la malla centrada en el ventilador

\begin{tabular}{llllll}
\hline $0.7,0.1$ & $0.1,0.1$ & $0.7,0.7$ & $0.5,0.2$ & $0.0,4.3$ & $0.0,0.0$ \\
$0.7,0.2$ & $1.2,1.6$ & $1.0,2.4$ & $0.5,2.2$ & $2.4,2.5$ & $0.3,0.1$ \\
$0.5,0.4$ & $1.2,4.7$ & $0.2,0.4$ & $0.2,0.2$ & $2.0,0.8$ & $0.4,0.2$ \\
$0.7,0.4$ & $0.7,4.4$ & $0.3,0.6$ & $0.0,0.2$ & $0.4,1.0$ & 06.0 .7 \\
$0.4,0.1$ & $1.3,5.9$ & $0.5,0.8$ & $0.3,1.7$ & $0.5,0.5$ & $0.5,0.4$ \\
$0.7,0.2$ & $0.2,0.2$ & $0.8,0.8$ & $0.7,1.7$ & $0.4,0.7$ & $0.2,0.2$ \\
\hline
\end{tabular}

\section{Rejillas de evacuación}

De las mediciones de velocidad del viento en estas rejillas (Tabla 3), es claro que son casi inútiles y que sólo operan a un flujo insignificante cuando el vagón se encuentra prácticamente hermético. Sin embargo, debe mencionarse que no se logró localizar ninguna otra salida de aire.
Evaluaciones en condiciones de operación

Las mediciones se efectuaron en seis vagones de la línea 3 y en doce vagones de la línea 1 , (seis de trenes modernos y seis de trenes antiguos). En cada unidad se midieron las velocidades de viento a las alturas de la cabeza de los pasajeros en pie y sentados. Estas 


\section{Estimación de la ventilación y el ruido en el metro de la ciudad de México}

mediciones se efectuaron durante los recorridos normales del metro.

Velocidades de viento promedio en diferentes zonas, línea 3

La tabla 4 presenta las velocidades de viento promedio, tanto en la zona cercana al ventilador, como en la menos cercana, que en promedio apuntan un viento de $0.5 \mathrm{~m} / \mathrm{s}$ para los pasajeros en pie y de $0.35 \mathrm{~m} / \mathrm{s}$ para los que van sentados.
Los gradientes de viento son sumamente marcados y no puede garantizarse la existencia de patrones en ningún punto de la unidad. La excepción es la zona cercana a la pared entre las puertas y las ventanas; en esta zona la infiltración de aire por la ventanilla es máxima, lo cual favorece la comodidad, pero sólo beneficia a un reducido número de pasajeros. La tabla 5 y la figura 2 presentan los resultados de la velocidad de viento en esa zona.

Tabla 3. Velocidad de viento en las rejillas de succión y de descarga, vagón vacío en reposo

\begin{tabular}{ccc}
\hline Ventanillas & Rejillas succión & Rejillas evacuación \\
\hline cerradas & $1.6 \mathrm{~m} / \mathrm{s}$ & $0.2 \mathrm{~m} / \mathrm{s}$ \\
abiertas & $1.6-1.7$ & 0.0 \\
\hline
\end{tabular}

Tabla 4. Velocidad de viento vagón en movimiento con pasajeros, ventanillas abiertas, pasillo de vagón, línea 3

\begin{tabular}{cccc}
\hline Distancia al techo $(\mathrm{m})$ & Velocidad promedio $(\mathrm{m} / \mathrm{s})$ & Velocidad mínima $(\mathrm{m} / \mathrm{s})$ & Ruido $(\mathrm{dB})$ \\
\hline 0 & 6.2 & & 80 \\
0.35 & 1.1 & & 80 \\
Cabezas pasajeros de pie & $0.5 \pm 0.2$ & $0.1-0.2$ & 80 \\
Cabezas pasajeros sentados & $0.35 \pm 0.08$ & $0.1-0.3$ & 78 \\
\hline
\end{tabular}

Nov., $2002, \mathrm{~T}_{\text {ambiente }}=29.5-30.1^{\circ} \mathrm{C}$

Tabla 5. Velocidad de viento, tren en movimiento con pasajeros, ventanillas abiertas, zona de máxima infiltración de aire, línea 3

\begin{tabular}{cc}
\hline Distancia de la pared hacia el centro a la altura de los & Vasajeros de pie \\
\hline $10-20 \mathrm{~cm}$, puertas abiertas & 1.3 \\
$10-20 \mathrm{~cm}$, en movimiento & 2.3 \\
$20 \mathrm{~cm}$ de la ventana & 2.0 \\
$30 \mathrm{~cm}$ de la puerta & 1.3 \\
$60 \mathrm{~cm}$ de la puerta & 0.2 \\
\hline
\end{tabular}




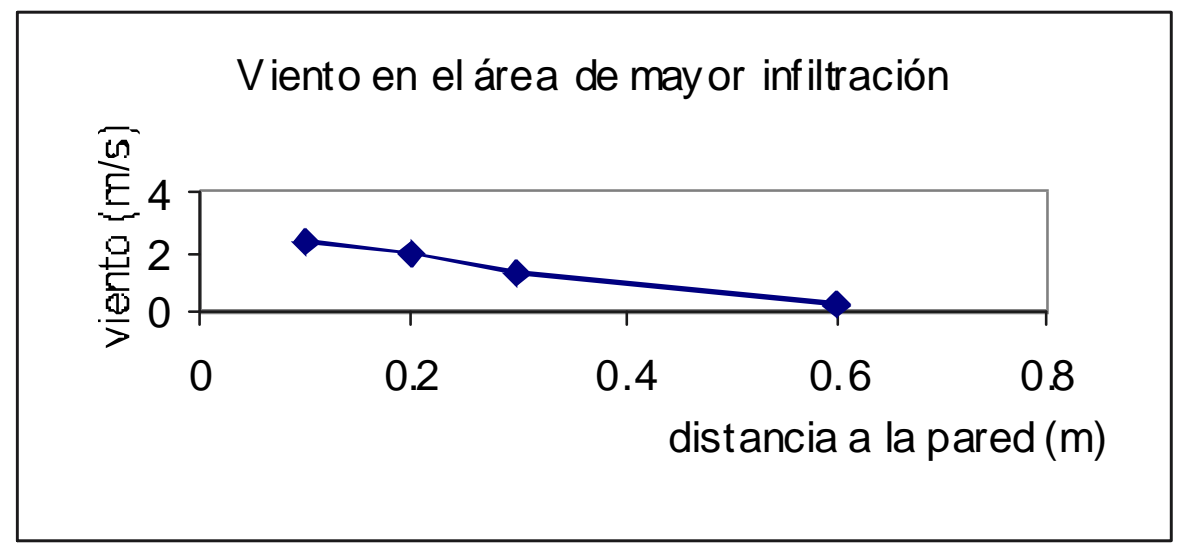

Figura 2

Rejillas de evacuación, línea 3

La mediciones con las ventanillas abiertas confirmaron su nula utilidad.

Velocidades de viento promedio en diferentes zonas, línea 1

Las tablas 6,7 y la figura 3 presentan los resultados de las velocidades de viento promedio a la altura de los pasajeros de pie y de los pasajeros sentados. Como se observa, para la primera la velocidad de viento (1.0 a 2.0 $\mathrm{m} / \mathrm{s}$ ) es considerablemente superior a la medida en los vagones de la línea 3. A la altura de los pasajeros sentados la velocidad de viento sigue resultando un tanto baja $(0.2-0.3$ $\mathrm{m} / \mathrm{s})$, pero también existe mejoría con respecto a la línea 3. En condiciones normales con ventanillas abiertas, la velocidad de viento en las rejillas de evacuación fue nula. No se hicieron mediciones con las ventanillas cerradas. En las rejillas de succión, la velocidad fue $0.4 \mathrm{~m} / \mathrm{s}$, (Tabla 8 ).

Tabla 6. Velocidad de viento, en movimiento con pasajeros, ventanillas abiertas, pasillo, línea 1 (trenes antiguos)

\begin{tabular}{ccc}
\hline Zona de medición & Velocidad promedio $(\mathrm{m} / \mathrm{s})$ entre el centro del ventilador $\mathrm{y} \pm 72 \mathrm{~cm}$ & Ruido promedio $(\mathrm{dB})$ \\
\hline Cabezas pasajeros de pie & 0.8 & $75-77$ \\
Cabezas pasajeros sentados & 0.4 & 77 \\
\hline
\end{tabular}

Enero $22,2002, \mathrm{~T}_{\text {ambiente }}=31.1^{\circ} \mathrm{C}$

Tabla 7. Velocidad de viento, en movimiento con pasajeros, ventanillas abiertas, pasillo, línea 1 (trenes modernos)

\begin{tabular}{ccc}
\hline Zona de medición & $\begin{array}{c}\text { Velocidad promedio }(\mathrm{m} / \mathrm{s}) \text { entre el centro } \\
\text { del ventilador } \mathrm{y} \pm 72 \mathrm{~cm}\end{array}$ & $\begin{array}{l}\text { Velocidad promedio }(\mathrm{m} / \mathrm{s} \text { ) a una distancia } \\
\text { entre } 72 \mathrm{~cm} \text { y } 1 \text { imdel centro del ventilador }\end{array}$ \\
\hline Cabezas pasajeros de pie & $1-1.2$ & $1.7-2.4$ \\
Cabezas pasajeros sentados & $0.25-0.3$ & --- \\
\hline
\end{tabular}

Enero $22,2002, \mathrm{~T}_{\text {ambiente }}=31.1^{\circ} \mathrm{C}$ 


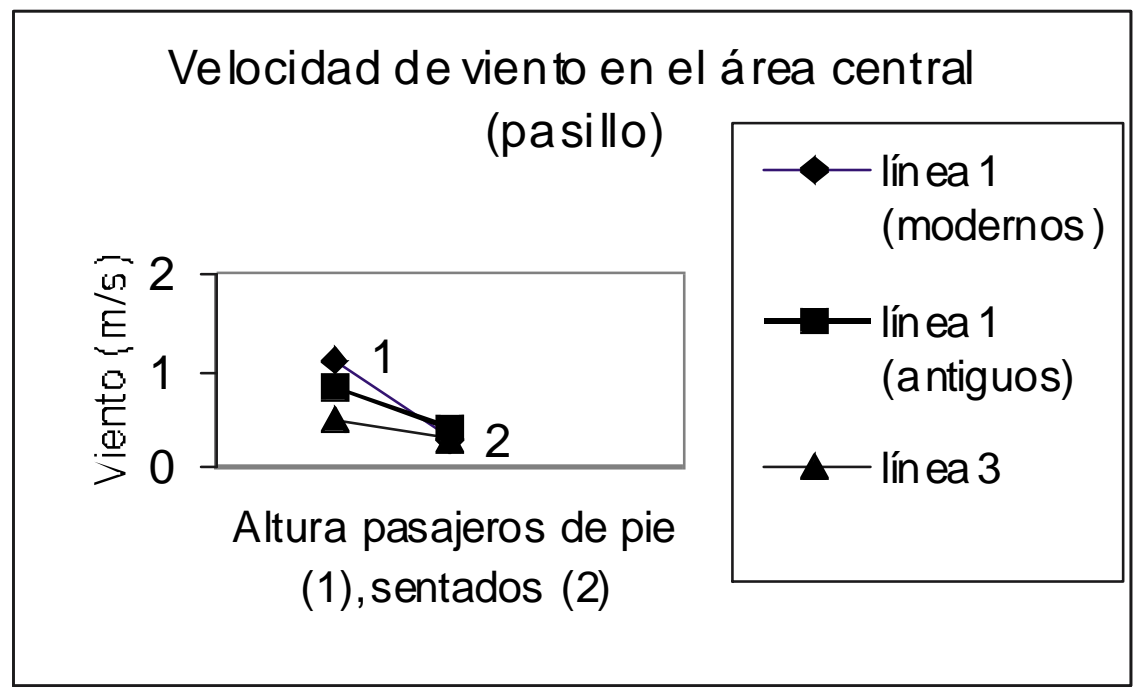

Figura 3

Tabla 8. Velocidad de viento, en movimiento con pasajeros, ventanillas abiertas, línea 1 (trenes modernos)

Rejillas de succión (tren estacionado)

Rejillas de evacuación

\section{Evaluación individual de los ventiladores}

Tanto en la línea 3 como en la 1 se evaluaron los siete ventiladores de un vagón escogido al azar. Aunque no se siguió estrictamente la norma, se aproximó lo suficiente para obtener una evaluación aceptable. La medición del gasto volumétrico de cada ventilador, se hizo con un ducto de cartón de sección transversal rectangular, de $0.58 \mathrm{~m} \times 0.50 \mathrm{~m}$ y $1.0 \mathrm{~m}$ de largo, el cual se instaló con cinta adhesiva en el techo. La sección transversal se dividió en 36 celdas, formando una malla de $6 \times 6$, y se midió la velocidad en el centro de cada celda.

La mayoría de los ventiladores modernos dio un gasto superior a los $1,600 \mathrm{~m}^{3} / \mathrm{h}$, con un total en el vagón superior a los $12,200 \mathrm{~m}^{3} / \mathrm{h}$; sin embargo, la distribución de aire no es la más conveniente. Por otro lado, en la mayoría de los ventiladores del vagón antiguo, el gasto fue bajo, con un total inferior a $4,500 \mathrm{~m} / \mathrm{h}$, incumpliendo con los valores de diseño, probablemente debido a las obstrucciones internas entre las rejillas y la salida del ventilador.

\section{Ruido}

Los valores de ruido en la salida del ventilador fueron, en la mayoría de los casos, cercanos a 90-100 dB, y a la altura de los pasajeros entre 75 y $85 \mathrm{~dB}$. El ruido de los ventiladores resulta molesto por lo monótono, no obstante, la diferencia de ruido entre los vagones con los ventiladores operando y aquellos con ventiladores apagados fue poco significativa. Por el contrario, la diferencia es notoria dependiendo de la zona del vagón en que se mida; por ejemplo, se midió una diferencia de $5 \mathrm{~dB}$ entre el centro y el extremo de los vagones. 
La norma que regula los límites máximos permisibles de exposición al ruido (Apéndice A) in dica que éste está dentro de lo permisible, aunque su valor es alto, particularmente para quienes deben permanecer cerca de los ventiladores, por lo que no es de aceptarse como satisfactorio para las condiciones de comodidad. Además, en algunos casos, aumentó hasta en siete $d B$ con la voz de algunos vendedores que ofrecían sus productos con voz sumamente alta y molesta.

\section{Observaciones en los vagones antiguos}

1. La aspiración del aire por los motoventiladores debe realizarse a través de las rejillas laterales colocadas en la parte superior de los vagones (arriba de las ventanillas), no obstante, debido a la obstrucción de filtros, estructuras y cables, parece presentar serias deficiencias.

2. La evacuación del aire no se pudo establecer, pues en condiciones normales las rejillas presentan un flujo nulo hacia el exterior y no se detectó flujo de salida por las ventanillas.

3. La operación de los ventiladores no proporciona ninguna comodidad a los pasajeros.

4. La velocidad de viento a la altura de los ocupantes sentados es prácticamente nula.

\section{Observaciones en los vagones modernos}

Se observó mayor y mejor ventilación que en los vagones antiguos. En condiciones de operación normal, la ventilación fue razonablemente aceptable, sin que se hayan detectado patrones de flujo en ninguno de los vagones en que se midió; además, se observaron grandes variaciones de viento a lo largo del vagón, y de vagón a vagón.

\section{Observaciones generales}

1. En la mayoría de los ventiladores, la velocidad de viento a la salida es asimétrica, y varía entre $0.1 \mathrm{~m} / \mathrm{s}$ y $2.3 \mathrm{~m} / \mathrm{s}$ llegando, excepcionalmente, hasta $6 \mathrm{~m} / \mathrm{s}$.

2. De los ventiladores observados, se estima que $80 \%$ presentó un desbalanceo apreciable y solamente un $20 \%$ operaba adecuadamente.

3. Se localizaron zonas de entrada de aire tanto por las rejillas como por las ventanillas; sin embargo, no se logró localizar ninguna zona de evacuación del aire, ya que no se observó salida de aire ni por las rejillas ni por las ventanillas.

4. Un importante ingreso de aire se debe a la circulación por efecto pistón a través de las ventanillas, cuando se tiene un tren circulando en sentido contrario. Esta contribución no fue evaluada.

5. Al abrir las puertas se tiene una sensación de frescura, a pesar de que la temperatura de la estación sea sólo uno o dos grados inferior a la del vagón. Esto ocurre debido a que el aire puede salir libremente, e indica que no es la entrada, sino la falta extracción y de circulación del aire lo que impide la ventilación adecuada.

6. A pesar de la alta temperatura ambiente, en un importante número de vagones y en trenes completos, los ventiladores estaban fuera de operación. Se observó que un promedio de dos de 
los siete ventiladores de cada vagón estaba fuera de servicio.

- En numerosas ocasiones, a pesar de la alta temperatura ambiente, la mayoría de las ventanillas permanecen cerradas sin que los pasajeros hagan ningún intento por abrirlas. Lo anterior puede deberse a que sólo unos pocos están dispuestos a hacer el esfuerzo para abrirlas o cerrarlas, 0 a que en la mayoría de las ocasiones éste resulta infructuoso por el funcionamiento defectuoso de las ventanillas. Además, también se observa que un importante número de pasajeros mantiene puesta su chamarra, saco, etc., a pesar del ambiente caluroso.

\section{Conclusiones}

En los trenes antiguos, la ventilación es prácticamente inútil, ya que los flujos de aire no llegan a los pasajeros. No obstante, las mejoras implantadas aún en los trenes modernos, la ventilación varia excesivamente de punto a punto y de vagón a vagón.

El mantenimiento del sistema debe ser costoso debido a que cada uno de los 42 ventiladores de cada tren debe hacerse individualmente, incluyendo la labor especializada de balanceo, que requiere tiempo, herramientas y experiencia. El mecanismo para subir/bajar las ventanillas es inadecuado. Se sugiere revisar tal situación para procurar un sistema efectivo.

Al parecer, el problema más importante no radica en la entrada de aire, sino en su extracción y circulación adecuadas. Siendo las rejillas de extracción prácticamente inútiles, se sugiere que para sistemas futuros, se efectúe un rediseño completo que asegure la extracción y circulación efectivas del aire en puntos estratégicos. El ruido no rebasa las normas, pero resulta molesto, y es mayormente atribuible al tren y no a los ventiladores; sin embargo, la frecuencia del ruido de éstos resulta muy desagradable. Otros sonidos particularmente desagradables, resultan del continuo vocear de los vendedores y cantantes que hacen del viaje en el metro una experiencia que nos hace expresar... "que bueno que ya me voy a bajar".

\section{Agradecimientos}

El autor agradece la valiosa ayuda de sus alumnos del curso de termodinámica que ayudaron a realizar la mediciones. De igual manera, a la licenciada Irma Hinojosa por las correcciones efectuadas a este trabajo.

\section{Apéndice $A$}

\section{Limites permisibles de exposición al ruido}

Este apéndice establece los límites máximos permisibles de exposición de los trabajadores a ruido estable, inestable 0 impulsivo durante el ejercicio de sus labores, en una jornada laboral de 8 horas, según la tabla A.1.

Tabla A.1 NOM-011-stps-2001

Límites máximos permisibles de exposición

\begin{tabular}{cc}
\hline NER & TMPE \\
\hline $90 \mathrm{~dB}(\mathrm{~A})$ & 8 horas \\
$93 \mathrm{~dB}(\mathrm{~A})$ & 4 horas \\
$96 \mathrm{~dB}(\mathrm{~A})$ & 2 horas \\
$99 \mathrm{~dB}(\mathrm{~A})$ & 1 hora \\
$102 \mathrm{~dB}(\mathrm{~A})$ & 30 minutos \\
$105 \mathrm{~dB}(\mathrm{~A})$ & 15 minutos \\
\hline
\end{tabular}


O.R. San Román-Gutiérrez

\section{Referencias}

ASHRAE Handbook Fundamentals. (1993). American Society of Heating and Air Conditioning Engi neers. Atlanta, USA. Carrier. (1987). M anual de aire acondi cionado. Carrier Corpo ration, Barcelona.

De Dear R.M. (2001). Atmospheric Science, Univer sity Sydney.
Kvisgard B. (1997). Thermal Comfort. Innova Air Instru ments A/S, Denmark [Brüel \& Kjaer].

\section{Bibliografía sugerida}

Hernández G. (1999). Fundamentos de aire acondicionado.

Pita E.G. (1994). A condicionamiento de aire. CECSA.

\section{Semblanza del autor}

O scar Rafael San Román-G utiérrez. Recibió el título de físico por la UNAM en 1969, obtuvo la maestría en ciencias y el doctorado en 1979 por la Universidad de Southampton, Inglaterra. De 1981 a 1993, fue investigador del Instituto de Ingeniería de la UNAM, en donde fungió como coordinador de mecánica, fluidos y térmica; asimismo, participó en proyectos y asesorías de ingeniería ambiental y térmica. Ha sido profesor de asignatura en la Universidad Iberoamericana y en el Tecnológico de Monterrey. Actualmente imparte cátedra en la Facultad de Ingeniería de la UNAM. 